\title{
Flavonoids: isolation, characterization, and health benefits
}

\author{
Abiche Ekalu ${ }^{1 *}$ (D) and James Dama Habila ${ }^{2}$
}

\begin{abstract}
Background: The unique and vast pharmacological activities of flavonoids have made them of research interest. This led to the use of various techniques to isolate and characterize them, intending to determine their potential health benefits.

Main text: The medicinal values of plant-based flavonoids that this literature review tends to summarize the pharmacological activities of these secondary metabolites from 22 selected plant families. The pharmacological shreds of evidence reported in the literature have proven that flavonoids have shown anti-cancer, anti-microbial, anti-oxidant, anti-inflammatory, anti-fungal, anti-ulcer, and anti-edematogenic activities. Out of these, 30\% showed to have anti-oxidant activity, key in protecting the body against free radicals. Besides, $18 \%$ of the references showed anti-microbial and anti-cancer activities. Further literature reports indicated that flavonoids from these families exhibited anti-inflammatory and anti-edematogenic (9\%), anti-viral and anti-ulcer (5\%), anti-fungal, anti-nociceptive, and anti-histamine (2\%).
\end{abstract}

Conclusion: The pharmacological activities of flavonoids from the various sources reviewed in this study show that the secondary metabolites could provide a scaffold for the development of potent anti-cancer drugs in the future.

Keywords: Flavonoids, Isolation, Characterization, Health benefits

\section{Background}

Flavonoids are phytochemicals responsible for the various colors in the seeds, flowers, fruits, leaves, and bark [1]. Flavonoids are a large class of natural aromatic compounds as there are reported to be the most common plants' phenolics $[2,3]$. Over the years, flavonoids have represented a vast percentage of phytochemicals from natural sources. It has been reported that more than 10 , 000 different classes of flavonoids have been found in kingdom Plantae [4-6]. Flavonoids are secondary metabolites found in organs of these plants with different functions $[7,8]$. Also, they have been reported from sources such as vegetables, wine, fruits, and beverages (tea) [9].

The chemical structures of flavonoids consist of C6C3-C6 [10] rings which correspond to two aromatic

\footnotetext{
* Correspondence: ekalumiracl@gmail.com

${ }^{1}$ Department of Chemistry, Faculty of Sciences, Nigerian Army College of Education, PMB, llorin, Kwara 1410, Nigeria

Full list of author information is available at the end of the article
}

rings $\mathrm{A}$ and $\mathrm{B}$ linked by three carbon atoms, which may lead to the formation of a third ring (C). Variations in this basic structure give the various subclasses of flavonoidal compounds. These are flavanones, isoflavones, flavones, flavanols (catechins), chalcones, flavonols, and anthocyanins [11-14]. The flavonoids present in the diet help in the prevention of cardiovascular disease [15]. The biological and oxidative properties of flavonoids are responsible for their anti-allergic, cardioprotective, antidiabetic, anti-inflammatory, anti-oxidative activity, and free radical scavenging capacity $[15,16]$. Also, flavonoids have been reported to exhibit anti-cancer activity [5]. Studies of flavonoids revealed that they are free radical scavengers and reducing agents [17]. Recent researches have focused on the health benefits of these secondary metabolites because of their preventive activity against diseases and anti-oxidative activity, anti-cancer activities, anti-viral activities, and anti-inflammatory $[18,19]$. Aside from the antioxidant activity of flavonoids, chelating properties [20],

\section{Springer Open}

(๑) The Author(s). 2020 Open Access This article is licensed under a Creative Commons Attribution 4.0 International License, which permits use, sharing, adaptation, distribution and reproduction in any medium or format, as long as you give appropriate credit to the original author(s) and the source, provide a link to the Creative Commons licence, and indicate if changes were made. The images or other third party material in this article are included in the article's Creative Commons licence, unless indicated otherwise in a credit line to the material. If material is not included in the article's Creative Commons licence and your intended use is not permitted by statutory regulation or exceeds the permitted use, you will need to obtain permission directly from the copyright holder. To view a copy of this licence, visit http://creativecommons.org/licenses/by/4.0/. 
their usage as anti-aging substances [21], capillary permeability, and inflammatory response [22], anti-bacterial and therapeutic [23], gastro-protective, and anti-diabetic activity [3] of these phenolics have been reported.

The protective effects such as the anti-inflammatory, anti-oxidant, anti-viral, and anti-tumor activity of flavonoids from natural sources are well documented [24]. The anti-carcinogenic activity of flavonoids has been linked to their anti-oxidant properties [25] which is due to the hydroxyl groups on the structure of the flavonoids [26]. Because of the importance of these phytochemicals, this review summarizes the isolation, characterization, and health benefits of these flavonoids taking into consideration those phytochemicals responsible for these activities. The health benefits reviewed were anti-cancer, anti-microbial, anti-oxidant, anti-inflammatory, antifungal, anti-ulcer, and anti-edematogenic activities.

\section{Main text}

\subsection{Methods}

The search was done by using keywords such as flavonoids on "science direct," "google scholar," "Scopus" database, and many journal sites. Journals employed in the search are Elsevier and Springer. Other search engines used as well as papers published between 2006 and 2019.

\section{Results}

\subsection{Flavonoids biosynthetic pathway}

Flavonoids or stilbenes biosynthetic pathway (Fig. 1) follow the extension of 4-hydroxycinnamoyl-CoA with three malonyl-CoA units, in which the poly- $\beta$-keto chain folded in different ways, via Aldo or Claisen reactions. Stilbene synthase and chalcone synthase (enzymes) couple the three malonyl-CoA with cinnamoyl-CoA unit to give chalcones or stilbene. Chalcones are precursors for the vast range of flavonoids and their derivatives found in plants. The nucleophilic attack (Michael type) of a phenolic group on $\alpha, \beta$-unsaturated ketone forms a six-membered heterocyclic ring such as naringenin. This isomerization reaction in an acid condition favors the flavanone while in basic condition, the chalcone [27]. Flavanones then give rise to variants of flavonoids such as flavonols, flavones, anthocyanidins, and catechins as shown in Fig. 1

\subsection{Sources and classification of flavonoids}

The various sources of flavonoids have been reported [28] and are given in Table 1 while their classification [29] is shown in Table 2 showing the subclasses. Similarly, Fig. 2 showed the basic skeleton of flavonoids and their various classes.

\subsection{Isolation of flavonoids}

The isolation of flavonoids involves various techniques available to natural products researchers that have simplified their isolation from crude extracts. These techniques are column chromatography (CC), high-performance liquid chromatography (HPLC), high-speed counter-current chromatography (HSCCC), counter-current chromatography (CCC), open centrifugal preparative thin layer chromatography (CPTLC), preparative thin-layer chromatography (PTLC), medium pressure liquid chromatography (MPLC), and high-pressure preparative liquid chromatography (HPLC) $[29,30]$. The details of the extraction, steps, and the solvents used for the structural elucidation and characterization of the flavonoids summarized in this review are shown in Table 5.

\subsection{Techniques used to elucidate the structure of flavonoids}

Natural products researchers use the following spectroscopic techniques to elucidate the structure of flavonoids. These are infrared spectroscopy (IR), nuclear magnetic resonance (NMR), ultra-violet spectrophotometry (UV), mass spectrometry (MS), and physical properties as electronic circular dichroism (ECD), melting point (m.pt), and specific rotation power $\left([\alpha]_{D}^{T}\right)$ for flavonoids with a stereocenter for ECD and $[\alpha] D$. Flavonoids have unique chemical shifts. These characteristic chemical shifts make it easier to characterize them. The characteristic chemical shift values reported [29] for some flavonoid classes are given (Table 3) and the UV absorption ranges for these flavonoids are shown (Table 4).

The isolation, characterization, and health benefits of these flavonoids are represented (Scheme 1).

\section{Discussion}

\subsection{Health benefits of flavonoids}

Extensive biological studies of flavonoids have revealed their health benefits including disease prevention $[5,72]$. They have exhibited anti-oxidant, anti-inflammatory, antibacterial, and anti-viral activities [73], anti-oxidant [74], anti-allergic anti-carcinogenic properties [75]. The protective effects of flavonoids have been reported as they help to reduce oxidative stress in the body. The cholesterollowering activity, anti-cancer, anti-oxidant of myricetin, tricin, apigenin, luteolin, quercetin, and isorhamnetin has been reported [24]. The anti-viral, anti-bacterial, anticancer, cardioprotective, and anti-inflammatory activity [76], as chelating agents and, are strong topoisomerase inhibitors [24] anti-aggregational, anti-atherosclerotic, and detoxification activities [77] of various flavonoids have been reported. These biological activities depend to a larger extent on the hydroxyl group in the flavonoids [78]. Quercetin has reduced the risk of cancer, eye diseases, arthritis, and allergic disorders [9]. The decreased risk of cardiovascular disease by proanthocyanins and flavone-3-ols has been reported [23]. The techniques used for the isolation, 


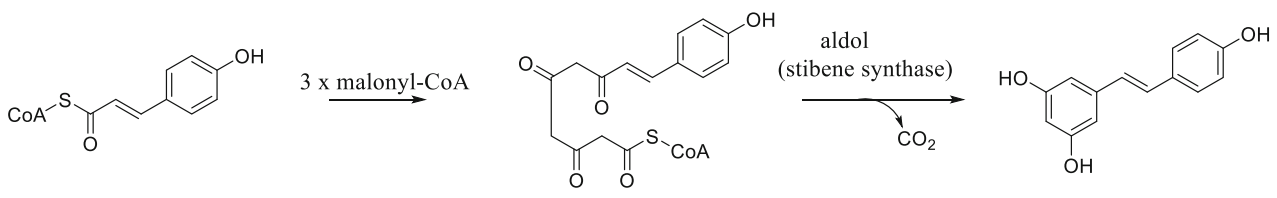

4-hydoxycinnamoyl-CoA

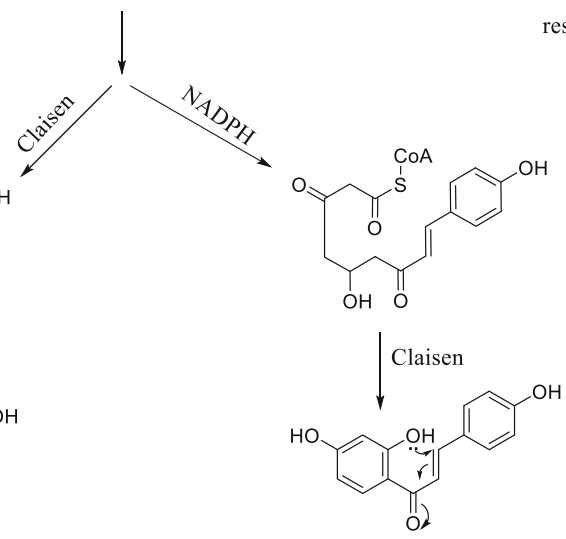

resveratrol<smiles>O=C(O)c1c(O)cc(O)cc1O</smiles>

isoliquiritigenin<smiles>O=c1cc(-c2ccc(O)cc2)oc2cc(O)ccc12</smiles><smiles>Cc1cc(C2CC(=O)c3c(O)cc(O)cc3O2)ccc1Cl</smiles>

$\mathrm{R}=\mathrm{H}$, naringenin

$\mathrm{R}=\mathrm{OH}$, erioictyol<smiles>[O]</smiles>

2-oxoglutarate

$\checkmark$<smiles>[R]c1cc(-c2cc(=O)c3c(O)cc(O)cc3o2)ccc1O</smiles>

$\mathrm{R}=\mathrm{H}$, apigenin $\mathrm{R}=\mathrm{OH}$, luteolin

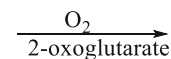

$\underset{\text { 2-oxoglutarate }}{\stackrel{\mathrm{O}_{2}}{ }}$<smiles>[R]c1cc(C2Oc3cc(O)cc(O)c3C(=O)C(O)C2O)ccc1O</smiles>

$\mathrm{R}=\mathrm{H}$, dihydrokaempferol

$\mathrm{R}=\mathrm{OH}$, dihydroquercetin NADPH<smiles>[R]c1cc(C2Oc3cc(O)cc(O)c3C(O)C2O)ccc1O</smiles>

$\mathrm{R}=\mathrm{H}$, leucopelargonidin $\mathrm{R}=\mathrm{OH}$, leucocyanidin

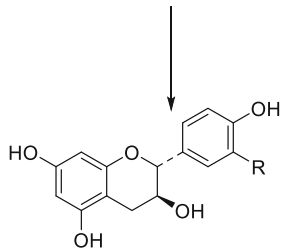

$\mathrm{R}=\mathrm{H}$, afzalechin $\mathrm{R}=\mathrm{OH},(+)$-catechin
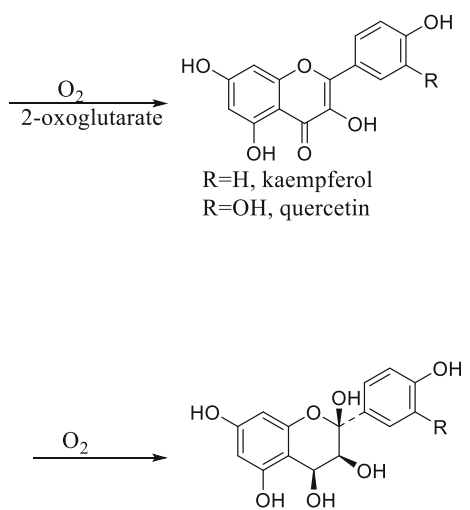

$\mathrm{R}=\mathrm{H}$, kaempferol $\mathrm{R}=\mathrm{OH}$, quercetin

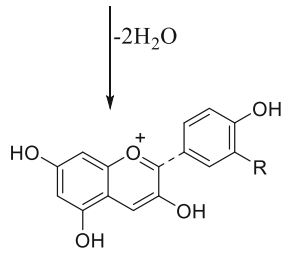

$\mathrm{R}=\mathrm{H}$, pelargonidin $\mathrm{R}=\mathrm{OH}$, cyanidin

Fig. 1 Biosynthetic pathway of flavonoid 
Table 1 Sources of flavonoids [28]

\begin{tabular}{ll}
\hline Flavonoids & Sources \\
\hline Flavonols & Ginger, broccoli, onions, leafy greens \\
Flavanols & Chocolate, red wine, black, and green tea \\
Flavonones & Oregano, celery, parsley \\
Flavanones & Citrus fruits, juices \\
Anthocyanidins & Red cabbage, grapes, berries, cherries \\
Isoflavonones & Milk, tofu, soy, tempeh \\
\hline
\end{tabular}

characterization, and the health benefits of these flavonoids are as shown in Table 5.

The pharmacological activities of the phytochemical constituents from 22 plant families reported in the literature as reviewed in Fig. 3 showed the percentages of these activities. Out of the references cited, $30 \%$ of the flavonoids showed anti-oxidant activity. Because of this vast anti-oxidant activity, flavonoids reduce aging by protecting the body against free radicals oxidation $[26,79]$.

\subsection{Anti-oxidant activity}

Anti-oxidants are compounds that slow or prevent oxidation in living cells. They act against the effects of free radicals. Flavonoids protect the body against reactive oxygen species. Chemically, flavonoids have hydroxyl groups and a highly conjugated $\pi$-electron system, which allows them to act as free radical scavengers [80]. Antioxidant activity of flavonoids [16, 20, 33, 76, 81], chelating properties [20], makes them acts as protective agents against free radicals $[26,79]$. In the body, anti-oxidants protect the human body from free radicals oxidation [17] thereby retarding the progress of many chronic diseases. Epicatechin, epigallocatechin, and gallocatechin have exhibited anti-oxidant activity [31].

Naringenin has shown anti-oxidant, anti-diabetic, antiatherogenic, anti-depressant, immunomodulatory, antitumor, antiinflammatory, and hypolipidaemic, activity [12]. Catechin, epicatechin, rutin, quercetin, and naringin have been reported for anti-oxidant activity against free radicals [32]. The anti-oxidant activity of spectaflovoside A, kaempferol-3-O-(2' ',3"'-di-O-acetyl)- $\alpha$-L- rhamnopyranoside, kaempferol-3-O(3' $3^{\prime}, 4^{\prime \prime}$-di-O-acetyl)$\alpha$-L-rhamnopyranoside, kaempferol-3-O- (2' ',4"'-di-Oacetyl)- $\alpha$-L-rhamnopyranoside, kaempferol, and kaempferol-3-O-(4' '-Oacetyl)- $\alpha$-L-rhamnopyranoside were documented [36]. The anti-oxidant activity of flavonoids extracted from Phlomis bovei De Noé [82], quercetin, taxifolin, catechin, and galangin, anthocyanidin, kaempferol, catechins, and catechin gallate esters have been reported [83]. Quercetin, anthocyanidin and kaempferol, catechins, and catechin gallate esters are effective anti-oxidants against free radicals. Quercetin had showed excellent in vitro anti-oxidant capacity [59]. Quercimeritrin, scutellarein, and rutin isolated from $C$. angustifolia showed strong anti-oxidant activity against oxidative stress [54]. The phytochemical investigation of the ethanol extract of Ximenia parviflora Benth. Var led to the isolation of quercetin, kaempferol, and apigenin with anti-oxidant activity. Similarly, the naringenin, quercetin, and kaempferol isolated from the ethanolic extract of Viscum album L showed anti-oxidant activity [62]. Catechins have been reported for its protection against oxidative stress, cancer, and cardiovascular disorder [84]. In Fig. 4, the chemical structures of flavonoids reported for their benefits are as shown.

\subsection{Anti-microbial activity}

The broth microdilution assay of spectaflovoside $\mathrm{A}$, kaempferol-3-O-(2' ', 3' '-di-O-acetyl)- $\alpha$-L-rhamnopyranoside, kaempferol-3-O-(3' ',4' '-di-O-acetyl)- $\alpha$-L-rhamnopyranoside, kaempferol-3-O-(4' '-Oacetyl)- $\alpha$-Lrhamnopyranoside, kaempferol, and kaempferol-3-O-(2' ',4' '-di-O-acetyl)- $\alpha$-L-rhamnopyranoside showed that these flavonoids exhibited remarkable anti-bacterial activities with a MIC values between $62.50 \mu \mathrm{g} / \mathrm{mL}$ and 500 $\mu \mathrm{g} / \mathrm{mL}$ against $E$. coli, K. pneumoniae, S. aureus, and $B$. cereus [36]. In another study, luteolin, 3,5-dihydroxy-6,7, 8,4'tetramethoxyflavone, apigenin, 3,5-dihydroxy-6,7,8trimethoxyflavone, apigenin 7-O-glucoside, apigenin $4{ }^{\prime}$ O-glucoside, kaempferol 3-O-glucoside, luteolin 4'-Oglucoside, luteolin 4',7-O-diglucoside, kaempferol, kaempferol 7-O-glucoside, and quercetin 3-O-glucoside

Table 2 Classification of flavonoids [29]

\begin{tabular}{ll}
\hline Flavonoids & Subclasses \\
\hline Flavanones & Hesperidin, naringenin, naringin, eriodityol, hesperidin \\
Flavones & Galangin, apigenin, chrysin, rpoifolin, baicalein, nobiletin, tangeretin, luteolin, \\
Anthocyanins & Catechin, cyanidin, epicatechin, pelargonidin, epicatechin gallate (ECG), malvidin, delphinidin, epigallocatechin (EGC), \\
Flavanols & Gallocatechin, epigallocatechin gallate (EGCG) \\
Chalcones & Arbutin, phloretin, chalconaringenin \\
Flavonols & Quercetin, rutin, myricetin, kaempferol, morin, fisetin, isorhamnetin \\
Isoflavonoids & Genistin, glycitein, daidzin, genistein \\
\hline
\end{tabular}


<smiles></smiles><smiles>O=c1c(-c2ccc(O)cc2)coc2cc(O)cc(O)c12</smiles><smiles></smiles><smiles>O=C(/C=C/c1ccc(O)cc1)c1c(O)cc(O)cc1O</smiles><smiles>O=C(/C=C/c1ccc(O)cc1O)c1cc(O)c(O)cc1O</smiles>
daidzein<smiles>O=c1c(-c2ccc(O)cc2)coc2cc(O)ccc12</smiles>

basic skeleton<smiles>COc1ccc(C2Oc3cc(O)cc(O)c3C(=O)C(O)C2O)cc1O</smiles>

Fig. 2 The basic skeleton of flavonoids and their various classes

were reported for their vast anti-microbial activities against $P$. aeruginosa [38]. Apigenin and isoflavones exhibited anti-bacterial activity [16]. Genistein, kaempferol, naringenin, and catechin isolated from Brassica oleracea var. Capitata L. possessed anti-bacterial activity against E. coli and S. aureus [52].

The flavonoids, 3-hydroxy flavone derivatives, and 3-methyl flavanone showed activity against Gram +ve bacteria [75]. Scandenone, kaempferol-3,7-O- $\alpha$-L-dirhamnoside tiliroside, quercetin-3,7-O- $\alpha$-L-dirhamnoside showed anti-microbial activity [43]. 7-Methoxy-3, 3',4',6-tetrahydroxyflavone, 3,3',4', 7-tetrahydroxyflavone (fisetin ), naringenin, 2',7-dihydroxy-4', 5 '-dimethoxyisoflavon, 3 '-hydroxydaidzein and xenognosin $B$ exhibited the anti-bacterial activities against B. subtili ATCC 6633, S. aureus ATCC25932, and B. cereus ATCC7064 [48]. The 2',4'-dihydroxy-4methoxy-3'-prenyldihydrochalcone, 4hydroxyonchocarpin, isobavachalcone, 2',4'-dihydroxy-3,4-(2' ',2' 'dimethylchromeno)-3'-prenyldihydrochalcone, 5,7-dihydroxy4'-methoxy-6-prenylflavanone, 5-hydroxy-6,7-(2, 2dimethylchromano)-4' -methoxyflavanone, 4',5-dihydroxy-6,

Table 3 Characteristic chemical shifts for flavonoids [29]

\begin{tabular}{ll}
\hline Chemical shifts (ppm) & ${ }^{1} \mathrm{H}$ \\
\hline $2-3$ & $\mathrm{H}-3$ (flavanone, $\mathrm{CH}_{3}$ aromatic) \\
$4-6$ & $\mathrm{H}-2$ (dihydroflavonol, flavanone) \\
$6-8$ & $\mathrm{~A}$ and B ring protons \\
$8-8.5$ & $\mathrm{H}-2$ (isoflavone) \\
$12-14$ & $5-\mathrm{OH}$ when $\mathrm{C}=\mathrm{O}$ at C4 (DMSO d6) \\
Chemical shifts (ppm) & ${ }^{13} \mathrm{C}$ \\
$28-35$ & $\mathrm{C}-4$ (flavanol) \\
$40-80$ & Non-oxygenated (C-2,-C-3-flavanone/flavanol) \\
$90-125$ (with ortho/para oxygenation) & Non-oxygenated aromatic carbons \\
$125-135$ (para substitution) & Non-oxygenated aromatic carbons \\
$130-150$ (with ortho/para oxygenation) & Oxygenated aromatic carbons \\
$155-165$ (no ortho/para oxygenation) & Oxygenated aromatic carbons \\
$170-210$ & $\mathrm{C}=\mathrm{O}$ \\
\hline
\end{tabular}


Table 4 UV absorption ranges for flavonoids [29]

\begin{tabular}{lll}
\hline Absorption $1(\mathrm{~nm})$ & Absorption 2 $(\mathrm{nm})$ & Types \\
\hline $250-280$ & $310-350$ & Flavone \\
$250-280$ & $330-360$ & Flavonols (3-OH-substituted) \\
$250-280$ & $350-385$ & Flavonols (3-OH-free) \\
$245-275$ & $310-330$ shoulder & Isoflavone \\
& C. 320 peak & Isoflavones (5-deoxy-6,7-deoxygenated) \\
$275-295$ & $300-330$ shoulder & Flavonones and dihydroflavonols \\
$230-270$ & $340-390$ & Chalcones \\
$230-270$ & $380-430$ & Aurones \\
$270-280$ & $465-560$ & Anthocyanidins and Anthocyanins \\
\hline
\end{tabular}

7(2,2-dimethylchromeno)-2' -methoxy-8- $\gamma, \gamma$-dimethylallylflavone, artocarpin, pyranocycloartobiloxanthone A, and cycloheterophyllin isolated from Artocarpus lowii King and Artocarpus anisophyllus Miq showed activity against S. aureus, P. putida, B. cereus, E. coli, C. albicans, and C. glabrata [49].

\subsection{Anti-cancer activity}

The term cancer refers to a disease in which cells of a tissue undergo uncontrolled and often rapid proliferation [85]. This is also the loss of control of growth [86]. Alternative medicine has been used to treat cancer [87]

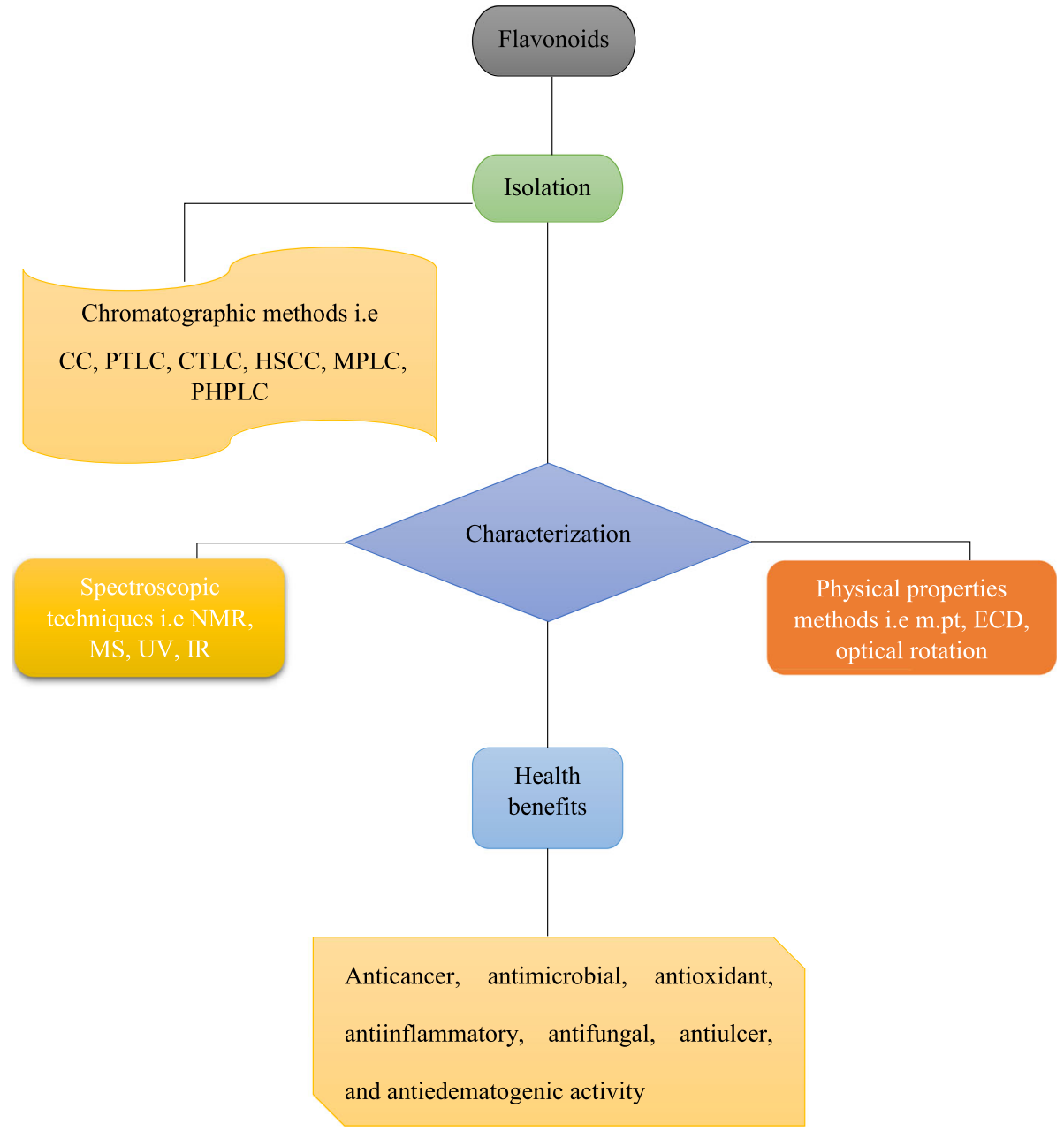

Scheme 1 Flavonoids: isolation, characterization, and health benefits 
Table 5 Isolation, characterization, and health benefits of flavonoids

\begin{tabular}{|c|c|c|c|c|c|c|}
\hline & $\begin{array}{l}\text { Isolation/ } \\
\text { characterization }\end{array}$ & $\begin{array}{l}\text { Solvents } \\
\text { used }\end{array}$ & Sources & Family & Health benefits & Ref. \\
\hline $\begin{array}{l}\text { Epicatechin, epigallocatechin, and } \\
\text { gallocatechin }\end{array}$ & HPLC & $\begin{array}{l}100 \% \\
\mathrm{H}_{2} \mathrm{O}\end{array}$ & Kombucha tea & & Anti-oxidant activity & [31] \\
\hline Naringenin & $\neq$ & $\neq$ & $\neq$ & $\neq$ & $\begin{array}{l}\text { Anti-oxidant activity, } \\
\text { hepatoprotective effects, anti- } \\
\text { inflammatory effects, anti- } \\
\text { carcinogenic effects, cardiovascular } \\
\text { effects, obesity, gastrointestinal effect, } \\
\text { naringenin enhances immunity }\end{array}$ & [12] \\
\hline $\begin{array}{l}\text { Catechin, epicatechin, rutin, } \\
\text { quercetin, naringin }\end{array}$ & UV-Vis & $\begin{array}{l}80 \% \\
\mathrm{MeOH} \\
\text { and } \\
100 \% \\
\text { acetone }\end{array}$ & $\begin{array}{l}\text { Cirsium } \\
\text { bulgaricum DC. }\end{array}$ & Asteraceae & $\begin{array}{l}\text { Anti-bacterial activity, anti-oxidant } \\
\text { activity }\end{array}$ & [32] \\
\hline Naringin, hesperidin, quercetin & HPLC & $\begin{array}{l}0.5 \mathrm{~g} \\
\mathrm{~kg}^{-1}\end{array}$ & Citrus fruits & Rutaceae & Anti-oxidant activity & [33] \\
\hline $\begin{array}{l}\text { Quercetin. kaempferol, myricetin, } \\
\text { luteolin, apigenin }\end{array}$ & HP2O & $\begin{array}{l}70 \% \\
\mathrm{EtOH}\end{array}$ & $\begin{array}{l}\text { Pluchea indica } \\
\text { (Linn.) Less }\end{array}$ & Asteraceae & $\begin{array}{l}\text { Anti-inflammatory, anti-nociceptive, } \\
\text { anti-inflammation activity }\end{array}$ & $\begin{array}{l}{[34,} \\
35]\end{array}$ \\
\hline $\begin{array}{l}\text { Spectaflovoside A, kaempferol-3-O-(2' } \\
\text { ',3"-di-O-acetyl)-a-L-rhamnopyrano- }\end{array}$ & NMR & $\begin{array}{l}\text { DCM: } \\
\text { EtOAC }\end{array}$ & $\begin{array}{l}\text { Zingiber } \\
\text { spectabile Griff }\end{array}$ & Zingiberaceae & $\begin{array}{l}\text { Anti-oxidant activity, anti-bacterial } \\
\text { activity }\end{array}$ & [36] \\
\hline
\end{tabular}

side, kaempferol-3-O-(3" 4"-di-O-

acetyl)-a-L-rhamnopyranoside,

kaempferol-3-O-(4"-Oacetyl)-a-L-

rhamnopyranoside and kaempferol,

kaempferol-3-O-(2",4"-di-O-acetyl)-a-

L-rhamnopyranoside

Quercetin glycosides, catechins

luteolin, apigenin, 3,5-dihydroxy-6,7,

8-trimethoxyflavone, kaempferol, 3,5-

dihydroxy-6,7,8,4'

tetramethoxyflavone, apigenin 4'-O-

glucoside, apigenin 7-O-glucoside,

luteolin 4'-O-glucoside, kaempferol 3-

O-glucoside, kaempferol 7-O-

glucoside, luteolin 4',7-O-diglucoside

and quercetin 3-O-glucoside

Quercetin, morin-3-O-lyxoside, quercetin-3-O-arabinoside, morin-3O-arabinoside

Rutin, quercetrin, quercetin, naringin, hesperidin, hespertin, kaempferol, apigenin, narengenin, 7-OH flavone

Quercetin, rutin, and naringenin

$\begin{array}{lll}\text { HPLC } & \mathrm{MeOH} & \text { Apple cultivars } \\ \text { PTLC and NMR } & 95 \% & \begin{array}{l}\text { Helichrysum } \\ \text { chasmolycium } \\ \text { P.H Davis }\end{array} \\ & & \end{array}$

Rosaceae

Asteraceae P.H Davis

chromatography

Tiliroside, scandenone, kaempferol-3, 7-O-a-L-dirhamnoside quercetin-3,7O-a-L-dirhamnoside

Quercetin

\begin{tabular}{|c|c|c|c|c|}
\hline HPLC & $\begin{array}{l}62.5 \% \\
\mathrm{MeOH}\end{array}$ & $\begin{array}{l}\text { Colvillea } \\
\text { racemosa }\end{array}$ & Caesalpinioideae & $\begin{array}{l}\text { Anti-microbial activity, anti-oxidant } \\
\text { activity, }\end{array}$ \\
\hline LC-ESI/MS & $\begin{array}{l}\text { EtOH } 70 \\
\%(v / v)\end{array}$ & $\begin{array}{l}\text { Raphanus sativus } \\
\text { L. }\end{array}$ & Brassicaceae & $\begin{array}{l}\text { Anti-microbial, anti-oxidant, anti- } \\
\text { histamine and anti-inflammatory } \\
\text { activity }\end{array}$ \\
\hline $\begin{array}{l}\text { MPLC, NMR and } \\
\text { MS }\end{array}$ & $\begin{array}{l}\text { EtOAc: } \\
\text { MeOH:n- } \\
\text { butanol }\end{array}$ & $\begin{array}{l}\text { Galium } \\
\text { fissurense, Viscum } \\
\text { album ssp. } \\
\text { album and } \\
\text { Cirsium } \\
\text { hypoleucum }\end{array}$ & $\begin{array}{l}\text { Rubiaceae, } \\
\text { Santalaceae and } \\
\text { Asteraceae }\end{array}$ & Anti-microbial activity \\
\hline PTLC, TLC, NMR & $\begin{array}{l}\mathrm{CHCl}_{3}: \\
\mathrm{EtOH}\end{array}$ & $\begin{array}{l}\text { Maclura } \\
\text { pomifera (Rafin.) } \\
\text { Schnider, } T \text {. } \\
\text { argentea, } \\
\text { Tilia argentea } \\
\text { Desf. ex DC. }\end{array}$ & $\begin{array}{l}\text { Moraceae, } \\
\text { Tiliaceae }\end{array}$ & $\begin{array}{l}\text { Anti-cancer, anti-bacterial, anti-fungal } \\
\text { activity, and anti-viral activity }\end{array}$ \\
\hline HPLC, FTIR, and & EtOAc & Aesculus indica & Sapindaceae & Anti-oxidant activity \\
\hline
\end{tabular}


Table 5 Isolation, characterization, and health benefits of flavonoids (Continued)

\begin{tabular}{|c|c|c|c|c|c|c|}
\hline & $\begin{array}{l}\text { Isolation/ } \\
\text { characterization }\end{array}$ & $\begin{array}{l}\text { Solvents } \\
\text { used }\end{array}$ & Sources & Family & Health benefits & Ref. \\
\hline Luteolin-7-O-glucoside & $\begin{array}{l}\text { TLC, HPLC-TOF/ } \\
\text { MS and FT-IR }\end{array}$ & $\begin{array}{l}\text { MeOH: } \\
\text { DCM (1: } \\
22 ; \mathrm{v} / \mathrm{v})\end{array}$ & $\begin{array}{l}\text { Tanacetum } \\
\text { abrotanifolium } \\
\text { (L.) Druce }\end{array}$ & Asteraceae & $\begin{array}{l}\text { Anti-cancer, anti-microbial, and anti- } \\
\text { oxidant activity }\end{array}$ & [45] \\
\hline Quercetin & TLC and HPLC & $\mathrm{EtOH}$ & $\begin{array}{l}\text { Nicotiana } \\
\text { tabacum }\end{array}$ & Solanaceae & Anti-oxidant activity & [46] \\
\hline $\begin{array}{l}\text { 7-methoxyflavanone, 3-acetoxy-4',5- } \\
\text { dihydroxy-and naringenin }\end{array}$ & $\begin{array}{l}\text { HPLC, NMR and } \\
\text { (HR)-EI-MS }\end{array}$ & $\begin{array}{l}\text { n-hex: } \\
\text { EtOAct }\end{array}$ & $\begin{array}{l}\text { Baccharis } \\
\text { dracunculifolia }\end{array}$ & Asteraceae & Anti-cancer activity & {$[47]$} \\
\hline $\begin{array}{l}\text { 2',7-dihydroxy-4',5'- } \\
\text { dimethoxyisoflavon, } \\
\text { 3'--hydroxydaidzein, 7-methoxy-3, 3', } \\
\text { 4',6-tetrahydroxyflavone } \\
\text { 3,3', } \\
\text { naringenin, and xenognosin B }\end{array}$ & $\begin{array}{l}\text { prep TLC, NMR, } \\
\text { MS, IR, m.pt and } \\
\text { optical rotation }\end{array}$ & $\begin{array}{l}1 / 3 \\
\text { EtOAC }\end{array}$ & $\begin{array}{l}\text { Boesenbergia } \\
\text { rotunda (L.) } \\
\text { Mansf. }\end{array}$ & $\begin{array}{l}\text { Streptomyces } \\
\text { sp. }\end{array}$ & Anti-bacterial activity & [48] \\
\hline $\begin{array}{l}\text { 2',4'-dihydroxy-4methoxy-3'- } \\
\text { prenyldihydrochalcone, 4- } \\
\text { hydroxyonchocarpin, isobavachal- } \\
\text { cone, 5,7-dihydroxy4'-methoxy-6-pre- } \\
\text { nylflavanone, 2',4'-dihydroxy-3,4-(2",2" } \\
\text { dimethylchromeno)-3'-prenyldihydro- } \\
\text { chalcone, 5-hydroxy-6,7-(2,2dimethyl- } \\
\text { chromano)-4'-methoxyflavanone, 4',5- } \\
\text { dihydroxy-6,7(2,2-dimethylchro- } \\
\text { meno)-2'-methoxy-8-y,y-dimethylallyl- } \\
\text { flavone, artocarpin, } \\
\text { pyranocycloartobiloxanthone A, and } \\
\text { cycloheterophyllin }\end{array}$ & TLC and NMR & $\begin{array}{l}\text { n-hex: } \\
\text { MeOH: } \\
\text { DCM }\end{array}$ & $\begin{array}{l}\text { Artocarpus } \\
\text { anisophyllus Miq. } \\
\text { and Artocarpus } \\
\text { lowii King }\end{array}$ & Moraceae & Anti-microbial activity & [49] \\
\hline Apigenin & HPLC & $\begin{array}{l}\mathrm{MeOH}: \\
\mathrm{H}_{2} \mathrm{O}(80 \text { : } \\
20)\end{array}$ & $\begin{array}{l}\text { Cousinia } \\
\text { verbascifolia } \\
\text { Bunge }\end{array}$ & Asteraceae & Anti-cancer activity & {$[50]$} \\
\hline Luteolin & $\begin{array}{l}\text { VLC, PTLC and } \\
\text { NMR }\end{array}$ & $\begin{array}{l}\mathrm{CHCl}_{3}: \mathrm{n-} \\
\operatorname{hex}(4: 5)\end{array}$ & $\begin{array}{l}\text { Struchium } \\
\text { sparganophora } \\
\text { (Linn) Ktze }\end{array}$ & Asteraceae & Anti-cancer activity & [51] \\
\hline $\begin{array}{l}\text { Genistein, kaempherol, naringenin, } \\
\text { and catechin }\end{array}$ & LC-MS & $\begin{array}{l}80 \% \\
\mathrm{MeOH}\end{array}$ & $\begin{array}{l}\text { Brassica oleracea } \\
\text { var. Capitata L. }\end{array}$ & Brassicaceae & Anti-bacterial activity & {$[52]$} \\
\hline $\begin{array}{l}\text { Quercetin-3-O- } \beta \text {-d-glucuronide, } \\
\text { luteolin-7-O- } \beta \text {-glucopyranoside, } \\
\text { formononetin-7-O- } \beta \text {-D-glucoside }\end{array}$ & HPLC & $\begin{array}{l}\mathrm{EtOH}: \\
\mathrm{H}_{2} \mathrm{O}: \mathrm{HCl} \\
(50: 20: 8)\end{array}$ & Cassia Tora linn. & Fabaceae & Anti-oxidant and anti-cancer activity & [53] \\
\hline Quercimeritrin, scutellarein, and rutin & HPLC-MS, NMR & $\begin{array}{l}\mathrm{MeOH}, \\
\text { EtOH, } \\
\text { acetone, } \\
\text { and } \\
\text { EtOAc }\end{array}$ & $\begin{array}{l}\text { Cassia } \\
\text { angustifolia Vahl. }\end{array}$ & Caesalpiniaceae & $\begin{array}{l}\text { Anti-cancer, anti-oxidants, and anti- } \\
\text { microbial activity }\end{array}$ & [54] \\
\hline Luteolin & HPLC & $\begin{array}{l}\mathrm{CHCl}_{3}: \\
\mathrm{MeOH} \\
(19: 1, \mathrm{v} / \\
\mathrm{v})\end{array}$ & $\begin{array}{l}\text { Vitex negundo } \\
\text { Lin }\end{array}$ & Lamiaceae & Anti-tumor activity & {$[55]$} \\
\hline $\begin{array}{l}\text { 2', 5-dihydroxy-7-methoxyflavone, 2', } \\
\text { 5Vdihydroxy-7-methoxyflavanone }\end{array}$ & LC-MS, NMR & $\begin{array}{l}100 \% \\
\mathrm{MeOH}\end{array}$ & $\begin{array}{l}\text { Andrographis } \\
\text { glandulosa, }\end{array}$ & Acanthaceae, & Anti-cancer activity & {$[56]$} \\
\hline Kaempferol & NMR, MS & $\begin{array}{l}\mathrm{CHCl}_{3}: \\
\mathrm{MeOH}(9: \\
1,7: 3,1: 1 \\
3: 7 \text { and } \\
1: 4)\end{array}$ & $\begin{array}{l}\text { Ageratum } \\
\text { conyzoides L. }\end{array}$ & Asteraceae & Anti-cancer and anti-oxidant activity & {$[57]$} \\
\hline Pinostrobin & $\begin{array}{l}\text { VLC, TLC, NMR, } \\
\text { EIMS }\end{array}$ & $\begin{array}{l}\text { Hex: } \\
\text { EtOAc } \\
80: 20 \\
(\mathrm{VLC} ; \\
15 \mathrm{~cm} \\
\text { x } 16 \mathrm{~cm})\end{array}$ & $\begin{array}{l}\text { Cajanus cojan } \\
\text { Millsp. }\end{array}$ & Fabaceae & Anti-cancer activity & [58] \\
\hline
\end{tabular}


Table 5 Isolation, characterization, and health benefits of flavonoids (Continued)

\begin{tabular}{|c|c|c|c|c|c|c|}
\hline & $\begin{array}{l}\text { Isolation/ } \\
\text { characterization }\end{array}$ & $\begin{array}{l}\text { Solvents } \\
\text { used }\end{array}$ & Sources & Family & Health benefits & Ref. \\
\hline Cyanarodide & $\begin{array}{l}\text { CC, TLC,NMR, } \\
\text { GC-MS }\end{array}$ & $\begin{array}{l}\text { EtOH: } \\
\text { EtOAc }+\end{array}$ & H. chillensis & Asteraceae & Anti-cancer activity & [59] \\
\hline $\begin{array}{l}\text { Procyanidin A, procyanidin B, and } \\
\text { catechin/epicatechin }\end{array}$ & $\begin{array}{l}\text { LC-MS, HPLC } \\
\text { DAD-MS/MS }\end{array}$ & $\mathrm{H}_{2} \mathrm{O}$ & $\begin{array}{l}\text { Ximenia } \\
\text { americana L. }\end{array}$ & Olacaceae & Anti-ulcerogenic activity & [60] \\
\hline Quercetin, kaempferol, and apigenin & HPLC-DAD & $\begin{array}{l}\text { EtOH (50, } \\
80 \text {, and } \\
100 \%, \mathrm{v} / \\
\text { v) }\end{array}$ & $\begin{array}{l}\text { Ximenia } \\
\text { parviflora Benth. } \\
\text { var. }\end{array}$ & Olacaceae & Anti-oxidant activity & [61] \\
\hline $\begin{array}{l}\text { Naringenin, quercetin, and } \\
\text { kaempferol }\end{array}$ & HPLC & $\begin{array}{l}\text { EtOH } \\
70 \%(1: \\
10, w / v)\end{array}$ & Viscum album L. & Santalaceae & Anti-oxidant activity & [62] \\
\hline $\begin{array}{l}\text { Catechin, rutin, quercitrin, quercetin, } \\
\text { and kaempferol }\end{array}$ & HPLC-DAD & $\begin{array}{l}\mathrm{H}_{2} \mathrm{O} 100 \\
\% \mathrm{EtOH} \\
(1: 1)\end{array}$ & $\begin{array}{l}\text { Ximenia } \\
\text { americana L. }\end{array}$ & Olacaceae & Anti-edematogenic activity & [63] \\
\hline 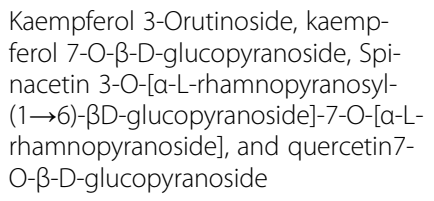 & $\begin{array}{l}\text { TLC, NMR and } \\
\text { ESI-MS }\end{array}$ & $\mathrm{EtOH}$ & Anvillea garcinii & Asteraceae & Anti-ulcer activity & [64] \\
\hline Hesperidin & $\begin{array}{l}\text { TLC, IR and } \\
\text { HPLC }\end{array}$ & $\mathrm{MeOH}$ & $\begin{array}{l}\text { Citrus sinensis (L.) } \\
\text { Osbeck }\end{array}$ & Rutaceae & Anti-ulcer activity & [65]. \\
\hline $\begin{array}{l}\text { Apigenin 4'-Oß-D-glucopyranoside, } \\
\text { isoquercetin, nicotiflorin and } \\
\text { apigenin 6-Ca-L-arabinopyranoside- } \\
\text { 8-C } \beta \text {-D-glucopyranoside }\end{array}$ & TLC, NMR & $\mathrm{EtOH}$ & Vicia sativa & Fabaceae & Anti-edematogenic activity $(P<0.05)$ & [66]. \\
\hline $\begin{array}{l}\text { cirsiliol, isorhamnetin 3-O-b-D-gluco- } \\
\text { side, chrysosplenol D, artemetin }\end{array}$ & TLC, NMR ,FT-IR & $\begin{array}{l}100 \% \\
\mathrm{MeOH}\end{array}$ & $\begin{array}{l}\text { Chrysanthemum } \\
\text { morifolim Ramat }\end{array}$ & Asteraceae & Anti-inflammatory activity & [67] \\
\hline Artemetin & CC and NMR & Acetone & $\begin{array}{l}\text { Cordia } \\
\text { curassavica DC }\end{array}$ & Boraginaceae & Anti-edematogenic activity & [68] \\
\hline 3-methoxy quercetin & TLC, NMR & $\begin{array}{l}\text { n- } \\
\text { butanol }\end{array}$ & $\begin{array}{l}\text { Garcinia kola } \\
\text { Heckel }\end{array}$ & Clusiaceae & Anti-inflammatory activity & [69] \\
\hline $\begin{array}{l}\text { Quercetin3-O- } \quad \text {-L-arabinopyranosyl } \\
(1 \rightarrow 2) \quad \text {-L-rhamnopyranoside }\end{array}$ & HPLC, NMR & $\begin{array}{l}\mathrm{H}_{2} \mathrm{O}: \\
\mathrm{EtOH}(1: \\
1) .\end{array}$ & $\begin{array}{l}\text { Kalanchoe } \\
\text { pinnata } \\
\text { (Lamarck) } \\
\text { Persoon }\end{array}$ & Crassulaceae & $\begin{array}{l}\text { Anti-inflammatory, anti-nociceptive, } \\
\text { and anti-edematogenic activity }\end{array}$ & [70] \\
\hline $\begin{array}{l}\text { 3,5-dihydro-7-methoxy } \\
\text { anthocynidines }\end{array}$ & PTLC, NMR & $\begin{array}{l}\mathrm{CHCl}_{3}: \\
\text { EtOAC } \\
(9: 1)\end{array}$ & $\begin{array}{l}\text { Monanthotaxis } \\
\text { littoralis }\end{array}$ & Annonaceae & Anti-fungal activity & [71] \\
\hline
\end{tabular}

*MPLC medium pressure liquid chromatography, LC-ESI/MS liquid chromatography electrospray ionization mass spectrometry, TLC thin layer chromatography, VLC vacuum liquid chromatography, LC-MS liquid chromatography-mass spectrometry, HPLC-MS high-performance liquid chromatography-mass spectrometry, EIMS electrospray ionization mass spectrometry, HPLC-DAD high-performance liquid chromatography with diode array detection, GC-MS gas chromatography-mass spectrometry, MIC minimum inhibitory concentration † Increasing polarity $\ddagger$ A review journal

and flavonoids, especially from plant sources, have not been left out. The pharmacological properties of flavonoids have made them useful alternatives to inhibit cell damage [73]. Flavonoids have been reported to be good anti-cancer therapeutics [88]. Quercetin particularly has been reported to be effective in the treatment of stomach, lung, prostate, and breast cancers [29]. Pradhan et al. 2015 reported the anti-tumor activity of quercetin without toxicity on the breast cancer cell, MCF-7 [89]. The anticancer activity of quercetin has been linked to the inhibition of the enzyme (DNA gyrase) [90]. Luteolin isolated from the leaves of Struchium sparganophora has caused cell death of melanoma and ovarian cancer cell lines [51]. Luteolin-7-O- $\beta$-glucopyranoside, formononetin-7-O- $\beta-D$ glucoside, and quercetin-3-O- $\beta$-d-glucuronide isolated from the leaves of Cassia tora linn were active against breast cancer (MCF7) [53]. Luteolin from the Vitex negundo Lin was an active anti-tumor agent [55]. In another study, the 2',5-dihydroxy-7-methoxyflavanone and 2', 5-dihydroxy-7-methoxyflavone isolated from Andrographis glandulosa were active against $\mathrm{HeLa}$, MIA PaCa, and U-8 [56]. Quercetin has been reported to induce 

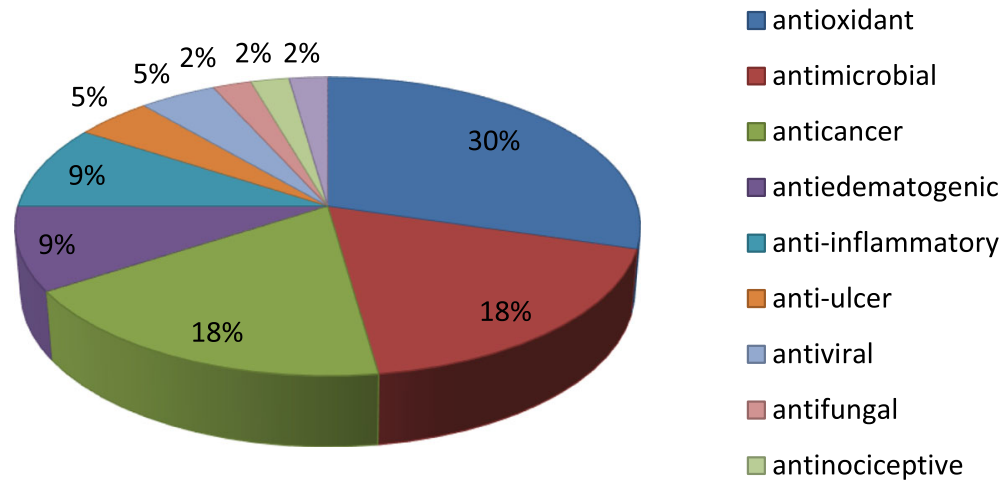

$\square$ Anti-histamine

Fig. 3 Representation of the activities of the flavonoids

cytotoxicity in cancer cells [91]. Kaempferol isolated from Ageratum conyzoides L. exhibited activity against lung cancer, gastric cancer, colon cancer, and glioma cancer [57]. Pinostrobin isolated from Cajanus cojan exhibited anti-leukemia activity [58]. The cynaroside isolated from H. chillensis was active against OVACAR-8, HCT-116, and SF-295 [59]. Nobiletin at $20 \mu \mathrm{M}$ inhibited human ovarian cancer [76].

Quercetin, kaempferol, myricetin, luteolin, and apigenin isolated from the aqueous and/or alcohol extracts of Khlu leaves exhibited anti-cancer activities [34]. Quercetin has shown anti-tumor activity [25]. Myricetin and quercetin inhibited mammalian TrxRs with $\mathrm{IC}_{50}$ values of 0.62 and $0.97 \mathrm{Mmol} / \mathrm{L}$, respectively [92]. Apigenin has been reported to arrest HT29 colon cancer [50]. Oncamex showed a strong anti-tumor effect against breast cancer while hesperetin was active against lung and carcinoma cancer cells [93]. Hesperidin and naringin isolated from the alcoholic extract of Colvillea racemosa were active against colon carcinoma cell lines (HCT116) [40]. Quercitin, morin, and myricetin have shown protective effects in the prevention of liver, cardiovascular diseases, and cancer [16].

\subsection{Anti-inflammatory}

Inflammation is a normal biological process in response to pathogen infection, tissue injury, or chemical irritation [16]. The anti-inflammatory properties of anthocyanins have been reported [81]. Previous in vitro studies of flavonoids showed inhibition against LPS-induced TNF- $\alpha$ production [94]. The report of the phytochemical investigation of Vicia sativa led to the isolation of apigenin 4'-O 3 -D-glucopyranoside, isoquercetin, nicotiflorin, and apigenin $6-\mathrm{C} \alpha-\mathrm{L}$-arabinopyranoside- $8-C \beta$-D-glucopyranoside that showed significant anti-edematogenic activity $(P<0.05)$ [66]. Isorhamnetin 3-O-b-D-glucoside, cirsiliol, chrysosplenol D, and artemetin isolated from Chrysanthemum morifolim Ramat inhibited the NO production in LPS-induced RAW
264.7 cells [67]. Daidzein, quercetin, genistein, and kaempferol have inhibited the production of both STAT-1 and NF-kB [95]. Similarly, the 3-methoxy quercetin isolated from Garcinia kola Heckel at concentrations (25 and 125 $\mu \mathrm{M})$ inhibited the production of TNF- $\alpha$ [69]. The quercetin 3-O- - -L-arabinopyranosyl $(1 \rightarrow 2)$ - -L-rhamnopyranoside isolated from Kalanchoe pinnata (Lamarck) Persoon showed anti-inflammatory activity [70]. At higher doses, flavonoids have shown a decrease in proliferation, CD14 surface marker, and NO production [96]. The flavonoids, artocarpanone $\mathrm{A}$, artocarpanone, and heteroflavanones $\mathrm{B}$ and $\mathrm{C}$ have shown a remarkable inhibitory effect on iNOS protein expression and NO production in RAW 264.7 cells [97].

\subsection{Anti-fungal activity}

The flavonoids, diglycosylflavones, flavonol-3-O-glycosides, and proanthocyanidins isolated from Ephedra alata showed anti-fungal activity [22], nobiletin and langeritin, and hesperidin exhibited strong and weak anti-fungal activities, respectively [2]. The flavonoid, baicalein, showed anti-candidal activity against $C$. tropicalis $170.06, C$. albicans ATCC 64550, and C. parapsilosis 153.07 with the $\mathrm{MIC}_{50}$ of 2.6, 26, and $13 \mu \mathrm{g} \mathrm{ml}^{-1}$, respectively [98]. The 3, 5-dihydroxy-7-methoxy anthocynidines isolated from Monanthotaxis littoralis was active against mycotoxigenic [71]. (-)-epicatechin-3-Ob-glucopyranoside, 6-phydroxybenzyltaxifolin-7-O-b-D-glucoside, 5-hydroxy-3(4-hydroxylphenyl) pyrano[3,2-g]chromene-4(8H)-one (-)-epicatechin(2(3,4-dihydroxyphenyl)-3,4-dihydro-2Hchromene-3,5,7-triol, and quercetin-3-O-a-glucopyranosyl-(1 2)-b-D-glucopyranoside isolated from Mangifera indica L exhibited anti-fungal activity [99].

\subsection{Anti-ulcer activity}

An ulcer is a disease of the alimentary tract caused by an inflamed break in the mucus lining membrane [100]. The anti-ulcer activity of quercetin in animals has been reported [72]. The phytochemical investigation of the 


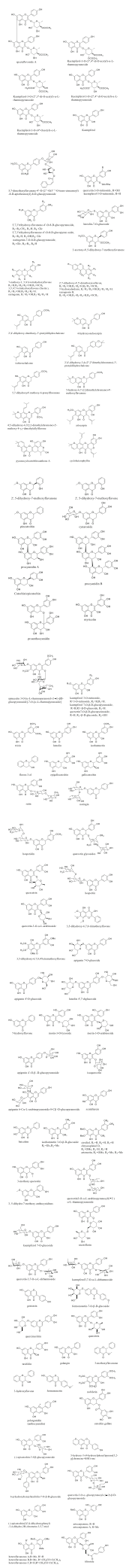

Fig. 4 Chemical structures of flavonoids with health benefits aqueous extract of Ximenia americana led to the isolation of procyanidins $B$ and $C$ as well as catechin/epicatechin which were active against acute gastric ulcer [60]. The spinacetin 3-O-[ $\alpha$-L-rhamnopyranosyl- $(1 \rightarrow 6)-\beta D-$ glucopyranoside]-7-O-[ $\alpha$-L-rhamnopyranoside], kaempferol-3-Orutinoside, kaempferol-7-O- $\beta$-D-glucopyranoside, and quercetin-7-O- $\beta$-D-glucopyranoside isolated from the ethanol extracts of Anvillea garcinii showed a powerful anti-ulcer [64]. The hesperidin isolated from Citrus sinensis showed anti-ulcer activity [65].

\subsection{Anti-edematogenic activity}

Catechin, rutin, quercitrin, quercetin, and kaempferol isolated from the $\mathrm{H}_{2} \mathrm{O} 100 \% \mathrm{EtOH}$ (1:1) of Ximenia americana $\mathrm{L}$ showed anti-edematogenic activity against ear edema in rat [63]. Artemetin isolated from Cordia curassavica DC exhibited remarkable anti-edematogenic activity [68].

\section{Conclusions}

Twenty-two members of the different families containing flavonoids studied for their health benefits confirmed the medicinal importance of these phytochemicals from these sources. The pharmacological pieces of evidence reported in the literature has proven that these flavonoids have shown anti-cancer, anti-microbial, anti-oxidant, anti-inflammatory, anti-fungal, anti-ulcer, and anti-edematogenic activity. Out of the references cited, $30 \%$ focused on the anti-oxidant activity of flavonoids, key in protecting the body against free radicals and oxidative stress. Also, $18 \%$ of the references showed anti-microbial and anti-cancer activities. Further literature reports indicated that flavonoids from these families exhibited anti-inflammatory and anti-edematogenic (9\%), antiviral and anti-ulcer (5\%), anti-fungal, anti-nociceptive, and anti-histaminice (2\%). The pharmacological activities of flavonoids from the various sources reviewed in this study show that the secondary metabolites could provide a scaffold for the development of potent anti-cancer drugs in the future.

\begin{abstract}
Abbreviations
MPLC: Medium pressure liquid chromatography; LC-ESI/MS: Liquid chromatography electrospray ionization mass spectrometry; TLC: Thin layer chromatography; VLC: Vacuum liquid chromatography; LC-MS: Liquid chromatography-mass spectrometry; HPLC-MS: High-performance liquid chromatography-mass spectrometry; EIMS: Electrospray ionization mass spectrometry; HPLC-DAD: High-performance liquid chromatography with diode array detection; GC-MS: Gas chromatography-mass spectrometry
\end{abstract}

\section{Acknowledgements}

The authors acknowledge the efforts of Dr. Eduard Mas Claret, a research fellow at the Department of Chemistry, University of Surrey, the UK, for reviewing the manuscript. 


\section{Funding}

None

\section{Availability of data and materials}

The search was by using keywords such as flavonoids. Other sources used are "science direct," "google scholar," "Scopus" database and many journal sites. Journals employed in the search are Elsevier and Springer. Other search engines used as well as papers published between 2006 and 2019

\section{Ethics approval and consent to participate}

Not applicable

\section{Consent for publication}

Not applicable

\section{Competing interests}

The authors declare that they have no competing interests.

\section{Author details}

'Department of Chemistry, Faculty of Sciences, Nigerian Army College of Education, PMB, Ilorin, Kwara 1410, Nigeria. ${ }^{2}$ Department of Chemistry, Faculty of Physical Sciences, Ahmadu Bello University, Zaria, Nigeria.

Received: 31 March 2020 Accepted: 30 June 2020

Published online: 29 September 2020

\section{References}

1. Sangeetha KSS, Umamaheswari S, Reddy CUM, Kalkura SN (2016) Flavonoids: Therapeutic potential of natural pharmacological agents. Int J Pharm Sci Res 7(10):3924-3930. https://doi.org/10.13040/IJPSR.0975-8232

2. Tapas A, Sakarkar D, Kakde R (2008) Flavonoids as Nutraceuticals: A Review. Trop J Pharm Res 7(3):1089-1099. https://doi.org/10.4314/tjpr.v7i3.14693

3. Datta N, Singanusong R, Chen SS, Yao LH, Jiang YM, Shi J, Tomás-Barberán FA (2004) Flavonoids in Food and Their Health Benefits. Plant Foods Hum Nutr 59:113-122

4. Rudrapal M, Chetia D (2016) Plant flavonoids as potential source of future antimalarial leads. Syst Rev Pharm 8(1):13-18. https://doi.org/10.5530/srp.2017.1.4

5. Kozłowska A, Szostak-Wegierek D (2014) Flavonoids_-food sources and health benefits. Rocz Panstw Zakl Hig 65(2):79-85

6. Weston LA, Mathesius U (2013) Flavonoids: their structure, biosynthesis and role in the rhizosphere, including allelopathy. J Chem Ecol 39:283-297. https://doi.org/10.1007/s10886-013-0248-5

7. Górniak I, Bartoszewski R, Króliczewski J (2019) Comprehensive review of antimicrobial activities of plant flavonoids. Phytochem Rev 18:241-272

8. Alzand Kl, Mohamed MA (2012) Flavonoids: Chemistry, Biochemistry and Antioxidant activity. J Pharm Res 5(8):4013-4020

9. Lakhanpal P, Rai DK (2007) Quercetin: A Versatile Flavonoid. Int J Med Update 2(2):22-37. https://doi.org/10.4314/ijmu.v2i2.39851

10. Sumira J, Abbas N (2013) Chemistry of Himalayan Phytochemicals. Stud Nat Prod Chem:1-15

11. Karak P (2019) Biological activities of flavonoids: an overview. Int J Pharm Sc Res 10(4):1567-1574. https://doi.org/10.13040/IJPSR.0975-8232

12. Venkateswara RP, Kiran SDVS, Rohini P, Bhagyasree P (2017) Flavonoid: a review on naringenin. J Pharmacogn Phytochem 6(5):2778-2783

13. Jaiswal N (2013) Protective effect of flavonoids in multiple sclerosis. J Sci Innov Res 2(3):509-511

14. Sülsen VP, Lizarraga E, Mamadalieva NZ, Lago JHG (2017) Potential of Terpenoids and Flavonoids from Asteraceae as Anti-Inflammatory, Antitumor, and Antiparasitic Agents. Evid Based Complement Altern Med 2017:6-8. https://doi.org/10.1155/2017/6196198

15. Egert S, Rimbach $\mathrm{G}$ (2011) Which sources of flavonoids: complex diets or dietary supplements? Am Soc Nutr Adv Nutr 2:8-14. https://doi.org/10.3945/ an. 110.0000268

16. Tiwari CS, Husain N (2017) Biological activities and role of flavonoids in human health - a review. Indian J Sci Res 12(2):193-196

17. Saxena M, Saxena J, Pradhan A (2012) Flavonoids and phenolic acids as antioxidants in plants and human health Int J Pharm Sci Rev Res 16(2):130-134

18. Ozcan T, Delikanli B, Yilmaz-Ersan L, Akpinar-Bayizit A (2014) Phenolics in human health. Int J Chem Eng Appl 5(5):393-396. https://doi.org/10.7763/ IJCEA.2014.V5.416
19. Lin G, Chan SSK, Chung HS, Li SL (2005) Chemistry and biological activities of naturally occurring phthalides. Stud Nat Prod Chem 32:611-669. https:// doi.org/10.1016/S1572-5995(05)80065-1

20. Heim KE, Tagliaferro AR, Bobilya DJ (2002) Flavonoid antioxidants: chemistry, metabolism and structure-activity relationships. J Nutr Biochem 13:572-584. https://doi.org/10.1016/S0955-2863(02)00208-5

21. Lumbiny BJ, Hui Z, Islam MA (2014) Antiaging, antioxidant flavonoids; synthesis, antimicrobial screening as well as 3D QSAR CoMFA models for the prediction of biological activity. J Asiat Soc Bangladesh Sci 39(2):191199. https://doi.org/10.3329/jasbs.v39i2.17856

22. Chebouat E, Dadamoussa B, Gharabli S, Gherraf N, Allaoui M, Cheriti A Lahham A, Zellagui A (2014) Assessment of antimicrobial activity of flavonoids extract from Ephedra alata. Der Pharm Lett 6(3):27-30

23. Hayat M, Abbas M, Munir F, Hayat MQ, Keyani R, Amir R (2017) Potential of plant flavonoids in pharmaceutics and nutraceutics. J Biomol Biochem 1(1):12-17

24. Singh NK, Rani M, Sharmila RT, Yadav AK (2017) Flavonoids in rice, their role in health benefits. MOJ Food Process Technol 4(3):96-99. https://doi.org/10. 15406/mojfpt.2017.04.00095

25. Kumar R, Vijayalakshmi S, Nadanasabapathi S (2017) Health Benefits of Quercetin. Def Life Sci J 2(2):142. https://doi.org/10.14429/dlsj.2.11359

26. Amić D, Davidović-Amić D, Drago B, Trinajstić N (2003) Structure-radical scavenging activity relationships of flavonoids dragan. Croat Chem Acta 76(1):55-61

27. Dewick PM (1998) Medicinal natural products: a biosynthetic approach, 1st edn. Wiley, pp 135-137

28. American Institute for Cancer Research (2015) Flavonoids in your foods: here's where to get them. www.aicr.org/wp-content/up

29. Awouafack MD, Tane P, Morita H (2017) Isolation and structure characterization of flavonoids. Intech open:45-59

30. Hostettmann K, Hostettmann M (1982) Isolation techniques for flavonoids. Flavonoids Springer Sci:1-18

31. Lobo RO, Dias FO, Shenoy CK (2017) Kombucha for healthy living: Evaluation of antioxidant potential and bioactive compounds. Int Food Res J 24(4):541-546

32. Karasakal A, Demirci AS, Demirok NT, Cabi E (2015) Antioxidant, antimicrobial activities and total flavonoid contents of Cirsium bulgaricum DC. leaf extracts. Marmara Pharm J 19:43-51. https://doi.org/10.12991/mpj. 2015198609

33. Iskender H, Yenice G, Dokumacioglu E, Kaynar O, Hayirli A, Kaya A (2016) The effects of dietary flavonoid supplementation on the antioxidant status of laying hens. Brazilian Journal of Poultry Science 18(4):663-668. https://doi. org/10.1590/1806-9061-2016-0356

34. Suriyaphan O (2014) Nutrition, health benefits and applications of Pluchea indica (L.) less leaves. Mahidol Univ J Pharm Sci 41(4):1-10

35. Traithip A (2005) Phytochemistry and Antioxidant Activity of Pluchea Indica, pp 4-6

36. Sivasothy $Y$, Sulaiman SF, Ooi KL, Ibrahim H, Awang K (2013) Antioxidant and antibacterial activities of flavonoids and curcuminoids from Zingiber spectabile Griff. Food Control 30:714-720. https://doi.org/10.1016/j.foodcont. 2012.09.012

37. Van Der Sluis AA, Dekker M, Jongen WMF, De Jager A (2003) Polyphenolic antioxidants in apples. Effect of storage conditions on four cultivars. Acta Hortic 600:533-540. https://doi.org/10.17660/ActaHortic.2003.600.80

38. Süzgeç-Selçuk S, Birteksöz AS (2011) Flavonoids of Helichrysum chasmolycicum and its antioxidant and antimicrobial activities. South African J Bot 77:170-174. https://doi.org/10.1016/j.sajb.2010.07.017

39. Rattanachaikunsopon P, Phumkhachorn P (2010) Contents and antibacterial activity of flavonoids extracted from leaves of Psidium guajava. J Med Plant Res 4(5):393-396

40. Shafei AA (2016) Qualitative and quantitative estimation of flavonoids and phenolic compounds and the biological activities of Colvillea racemosa cultivated in Egypt. Int J Pharmacogn Phytochem Res 8(5):836-840

41. Ngoc PTK, Nguyet NTM, Dao DTA (2017) Antimicrobial and antioxidant properties of the flavonoid extract from Raphanus sativus L. AIP Conf Proc 1878:020026. https://doi.org/10.1063/1.5000194

42. Özçelik B, Orhan DD, Özgen S, Ergun F (2008) Antimicrobial Activity of Flavonoids against Extended-Spectrum $\beta$-Lactamase (ESBL)-Producing Klebsiella pneumoniae. Trop J Pharm Res 7(4):1151-1157. https://doi.org/10. 4314/tjpr.v7i4.14701

43. Özçelik B, Orhan I, Toker G (2006) Antiviral and antimicrobial assessment of some selected flavonoids. Zeitschrift fur Naturforsch Sect C J Biosci 61:632-638 
44. Zahoor M, Shafiq S, Ullah H, Sadiq A, Ullah F (2018) Isolation of quercetin and mandelic acid from Aesculus indica fruit and their biological activities. BMC Biochem 19:1-14

45. Geçibesler HI (2017) In Vitro Biological Activity Studies on Tanacetum abrotanifolium (L.) Druce (Asteraceae). Anadolu Univ J Sci Technol A-Appl Sci Eng 18(2):439-455. https://doi.org/10.18038/aubtda.284496

46. Sharma Y, Nagar A, Srivastava NS, Bala K (2017) Antioxidant activity of polyphenolic flavonoid of stem of Nicotiana tabacum. Am J Drug Discov Dev 7(1):25-32. https://doi.org/10.3923/ajdd.2017.25.32

47. Fukuda M, Ohkoshi E, Makino M, Fujimoto Y (2006) Studies on the constituents of the leaves of Baccharis dracunculifolia (Asteraceae) and their cytotoxic activity. Chem Pharm Bull 54:1465-1468. https://doi.org/10.1248/cpb.54.1465

48. Taechowisan T, Chanaphat S, Ruensamran W, Phutdhawong WS (2014) Antibacterial activity of new flavonoids from Streptomyces sp. BT01; an endophyte in Boesenbergia rotunda (L.) mansf. J Appl Pharm Sci 4(4):8-13. https://doi.org/10.7324/JAPS.2014.40402

49. Jamil S, Lathiff SMA, Abdullah SA, Jemaon N, Sirat HM (2014) Antimicrobial flavonoids from Artocarpus Anisophyllus miq. and artocarpus lowii King. J Teknol 71(1):95-99. https://doi.org/10.11113/jt.v71.2699

50. Sajjadi SE, Ghanadian M, Haghighi M (2016) Isolation and identification of two phenolic compounds from a moderately cytotoxic fraction of Cousinia verbascifolia Bunge. Adv Biomed Res 6:1-5. https://doi.org/10.4103/22779175.190980

51. Kasim LS, Ferro V, Odukoya OA, Ukpo GE, Seidel V, Gray Al, Waigh R (2011) Cytotoxicity of isolated compounds from the extracts of Struchium sparganophora (Linn) Ktze asteraceae. Pak J Pharm Sci 24(4):475-478

52. Satish A, Farha SS, Urooj A (2018) Quantification of flavonoids by UPLC-MS and its antibacterial activity from Brassica oleracea var. Capitata L. GSC Biol Pharm Sci 5(1):109-114

53. Vijayalakshmi A, Masilamani K, Nagarajan E, Ravichandiran V (2015) In vitro antioxidant and anticancer activity of flavonoids from Cassia Tora linn. leaves against human breast carcinoma cell lines. Der Pharma Chem 7(9):122-129

54. Ahmed SI, Hayat MQ, Tahir M, Mansoor Q, Ismail M, Keck K, Bates RB (2016) Pharmacologically active flavonoids from the anticancer, antioxidant and antimicrobial extracts of Cassia angustifolia Vahl. BMC Complement Altern Med 16:1-9. https://doi.org/10.1186/s12906-016-1443-z

55. Chowdhury AR, Sharma S, Mandal S, Goswami A, Mukhopadhyay S, Majumder HK (2002) Luteolin, an emerging anti-cancer flavonoid, poisons eukaryotic DNA topoisomerase I. Biochem J 366:653-661. https://doi.org/10. 1042/BJ20020098

56. Cherukupalli N, Bhumireddy SR, Akella SSV, Sataniya A, Sripadi P, Khareedu VR, Vudem DR (2017) Phytochemical profiling and in vitro anticancer activity of purified flavonoids of Andrographis glandulosa. Planta Medica Int Open 4: 24-34. https://doi.org/10.1055/s-0043-105274

57. Tan NH, Akindahunsi AA, Zeng GZ, Zhang YM, Adebayo AH (2010) Anticancer and antiradical scavenging activity of Ageratum conyzoides L. (Asteraceae). Pharmacogn Mag 6(21):62. https://doi.org/10.4103/0973-1296.59968

58. Ashidi JS, Houghton PJ, Hylands PJ, Efferth T (2010) Ethnobotanical survey and cytotoxicity testing of plants of South-western Nigeria used to treat cancer, with isolation of cytotoxic constituents from Cajanus cajan Millsp. leaves. J Ethnopharmacol 128:501-512. https://doi.org/10.1016/j.jep.2010.01.009

59. da Silva ACN, do Nascimento RMC, do Nascimento RDC, Ferreira PMP, Pessoa C, Lima DJB, de Moraes Filho MOF, de Almeida RM, Ferreira SR, Fujiwara RT, do Nascimento AM (2019) In vitro activity evaluation of seven Brazilian Asteraceae against cancer cells and Leishmania amazonensis. South African J Bot 121:267-273. https://doi.org/10.1016/j.sajb.2018.11.008

60. Aragão TP, dos Prazeres LDKT, Brito SA, PJR N, Rolim LA, da Silva AJRG, Caldas GFR, Wanderley AG (2018) Contribution of secondary metabolites to the gastroprotective effect of aqueous extract of ximenia americana $\mathrm{L}$. (Olacaceae) stem bark in rats. Molecules 23:112. https://doi.org/10.3390/molecules23010112

61. Sánchez-Gutiérrez JA, Vázquez-Sánchez M, Álvarez-Bernal D, MaresQuiñones MD, Valiente-Banuet Jl, Medina-Medrano JR, Villar-Luna E (2018) Determination of phenolic compounds and the antioxidant capacity of Ximenia parviflora Benth. var. parviflora (Olacaceae) fruit by highperformance liquid chromatography with diode array detection. Anal Lett 51:1986-1998. https://doi.org/10.1080/00032719.2017.1404094

62. Simona loana Vicas DR and CS (2012) Antioxidant activity of European mistletoe (Viscum album), phytochemicals as nutraceuticals - global approaches to their role in nutrition and health,115-134

63. da Silva BAF, da Costa RHS, Fernandes CN, Leite LHI, Ribeiro-Filho J, Garcia TR, Coutinho HDM, Wanderley AG, de Menezes IRA (2018) HPLC profile and antiedematogenic activity of Ximenia americana L. (Olacaceae) in mice models of skin inflammation. Food Chem Toxicol 119:199-205. https://doi.org/10.1016/j.fct.2018.04.041

64. Perveen S, Fawzy GA, Al-Taweel MA, Orfali RS, Yusufoglu HS, Abdel-Kader MS, Al-Sabbagh RM (2018) Antiulcer activity of different extracts of Anvillea garcinii and isolation of two new secondary metabolites. Open Chem 16:437-445

65. Bigoniya P, Singh K (2014) Original article ulcer protective potential of standardized hesperidin, a citrus flavonoid isolated from Citrus sinensis. Brazilian J Pharmacogn 24:330-340

66. Gamal-Eldeen AM, Kawashty SA, Ibrahim LF, Shabana MM, El-Negoumy SI (2004) Evaluation of antioxidant, anti-inflammatory, and antinociceptive properties of aerial parts of Vicia sativa and its flavonoids. J Nat Remedies 4(1):81-96

67. Hu J, Ma W, Li N, Wang K (2017) Antioxidant and anti-inflammatory flavonoids from the flowers of Chuju, a medical cultivar of Chrysanthemum Morifolim Ramat. J Mex Chem Soc 61(4):282-289

68. Bayeux MC, Fernandes AT, Foglio MA, Carvalho JE (2002) Evaluation of the antiedematogenic activity of artemetin isolated from Cordia curassavica DC. Braz J Med Biol Res 35(10):1229-1232

69. Omeje EO, Nworu SC, Osadebe PO, Onugwu L, Maurya R, Okafor SN, Proksch P (2017) In- vitro anti-inflammatory activities of 3-methoxy quercetin isolated from Nigerian mistletoe parasitic on Garcinia kola Heckel, Clusiaceae. Trop J Pharm Res 16(5):1059-1067. https://doi.org/10.4314/tjpr.v16i5.13

70. Ferreira RT, Coutinho MAS, Malvar DC, Costa EA, Florentino IF, Costa SS, Vanderlinde FA (2014) Mechanisms underlying the antinociceptive, antiedematogenic, and anti-inflammatory activity of the main flavonoid from Kalanchoe pinnata. Evidence-Based Complement Altern Med 2014:1-8. https://doi.org/10.1155/2014/429256

71. Clara C, Matasyoh JC, Wagara IN, Nakavuma J (2014) Antifungal activity of flavonoids isolated from Monanthotaxis littoralis against mycotoxigenic fungi from maize. Am J Chem Appl 1(4):54-60

72. Rana AC, Gulliya B (2019) Chemistry and pharmacology of flavonoids- a review. Indian J Pharm Educ Res 53(1):130-134. https://doi.org/10.5530/ijper.53.1.3

73. Ruiz-Cruz S, Chaparro-Hernández S, Hernández-Ruiz KL, Cira-Chávez LA, Estrada-Alvarado MI, Gassos Ortega LE, José de Jesús Ornelas-Paz J, MAL M Flavonoids: Important Biocompounds in Food. Intech Open:354-369. https://doi.org/10.5772/67864

74. Procházková D, Boušová I, Wilhelmová N (2011) Antioxidant and prooxidant properties of flavonoids. Fitoterapia. 82:513-523. https://doi.org/10.1016/j. fitote.2011.01.018

75. Kamlesh KN, Sivakumar T, Afroze A (2017) Antimicrobial Activity of Flavone Analogues. J Appl Pharmacol 9(1):1-9. https://doi.org/10.21065/1920-4159. 1000232

76. Sandu M, Bîrsă LM, Bahrin LG (2017) Flavonoids - Small Molecules, High Hopes. Acta Chem lasi 25(1):6-23. https://doi.org/10.1515/achi-2017-0003

77. Majewska M, Skrzycki M, Podsiad M, Czeczot H (2011) Evaluation of antioxidant potential of flavonoids: An in vitro study. Acta Pol Pharm-Drug Res 68(4):611-615

78. Kumar S, Pandey AK (2013) Chemistry and Biological Activities of Flavonoids: An Overview. Sci World J:1-16. https://doi.org/10.1155/2013/ 162750

79. Russo D (2018) Pharmacognosy \& Natural products flavonoids and the structure-antioxidant activity relationship. J Pharmacogn Nat Prod 4(1):3031. https://doi.org/10.4172/2472-0992.1000e109

80. Martinez-Perez C, Ward C, Cook G, Mullen P, McPhail D, Harrison Dر, Langdon SP (2014) Novel flavonoids as anti-cancer agents: mechanisms of action and promise for their potential application in breast cancer. Biochem Soc Trans 42(4):1017-1023. https://doi.org/10.1042/BST20140073

81. Khan MAY, Mundasada SC, Ramadas D (2015) Antioxidant activity : root, leaves and fruits aqueous extracts of Muntingia Calabura. J Innov Pharm Bio Sci 2(4):363-368

82. Wafa N, Sofiane G, Mouhamed K (2016) The antioxidant and antimicrobial activities of flavonoids and tannins extracted from Phlomis bovei De Noé. Eur J Exp Biol 6(3):55-61

83. Nishigaki I, Peramaiyan R, Ramachandran V, Gnapathy E, Dhanapal S, Yutaka N (2010) Cytoprotective role of astaxanthin against glycated protein/iron chelate-induced toxicity in human umbilical vein endothelial cells. Phyther Res 24(9):54-59. https://doi.org/10.1002/ptr

84. Khanna A, Maurya PK (2012) Role of tea catechins in prevention of aging and age-related disorders. Tang [Humanitas Med] 2(1):2.1-2.11. https://doi.org/10.5667/tang.2011.0016 
85. Pawar SR, Jangam S, Waghmare S (2018) Anti-cancer herbal drugs: an overview. J Drug Deliv Ther 8(4):48-58

86. Elnour AM, Penech F, Mesaik AM (2018) Four selected sudanese medicinal plants induce anticancer and cytotoxic effects in prostate cancer cell line. Clin Med Biochem 3(2):3-6. https://doi.org/10.4172/2471-2663.1000134

87. Roy A, Ahuja S, Bharadvaja N (2017) A review on medicinal plants against cancer anticancer activity of medicinal plants. J Plant Sci Agric Res 2(1):1-5

88. Ragazzon PA, lley J, Missailidis S (2009) Structure-activity studies of the binding of the flavonoid scaffold to DNA. Anticancer Res 29:2285-2293

89. Pradhan D, Pradhan RK, Tripathy G, Pradhan S (2015) Inhibition of proteasome activity by the dietary flavonoid Quercetin associated with growth inhibition in cultured breast cancer cells and xenografts. J Young Pharm 7(3):225-233. https://doi.org/10.5530/jyp.2015.3.13

90. Cushnie TPT, Lamb AJ (2005) Antimicrobial activity of flavonoids. Int J Antimicrob Agents 26:343-356. https://doi.org/10.1016/i.jiantimicag.2005.09.002

91. Srivastava S, Somasagara RR, Hegde M, Nishana M, Tadi SK, Srivastava M, Choudhary B, Raghavan SC (2016) Quercetin, a natural flavonoid interacts with DNA, arrests cell cycle and causes tumor regression by activating mitochondrial pathway of apoptosis. Sci Rep 6:1-13. https://doi.org/10.1038/ srep24049

92. Lu J, Papp LV, Fang J, Rodriguez-Nieto S, Zhivotovsky B, Holmgren A (2006) Inhibition of mammalian thioredoxin reductase by some flavonoids: Implications for myricetin and quercetin anticancer activity. Cancer Res 66(8):4410-4418. https://doi.org/10.1158/0008-5472.CAN-05-3310

93. Stanisic D, Costa FA, Favaro FW, Tasic L, Seabra AB, Duran N (2018) Anticancer activities of hesperidin and hesperetin in vivo and their potentiality against bladder cancer. J Nanomed Nanotechnol 9(5). https://doi.org/10.4172/2157-7439.1000515

94. Ueda H, Yamazaki C, Yamazaki M (2004) A hydroxyl group of flavonoids affects oral anti-inflammatory activity and inhibition of systemic tumor necrosis factor- a production. Biosci Biotechnol Biochem 68(1):119-125. https://doi.org/10.1271/bbb.68.119

95. Nieminen R, Vuorela P, Heinonen M, Moilanen E (2007) Anti-inflammatory effects of flavonoids : genistein, kaempferol, quercetin, and daidzein inhibit STAT-1 and NF- $k$ B activations, whereas flavone, isorhamnetin, naringenin, and pelargonidin inhibit only NF-KB activation along with their inhibitory. Mediat Inflamm 2007:1-10. https://doi.org/10.1155/2007/45673

96. Gupta A, Chaphalkar SR (2016) Anti-inflammatory and immunosuppressive activities of flavonoids from medicinal plants. J Herb Med Pharmacol 5(3): 120-124

97. Bai-Luh W, Jing-Ru W, Pao-Hui C, Chi-Feng H, Jih-Pyang W, Lin C (2005) Antiinflammatory Flavonoids from Artocarpus heterophyllus and Artocarpus communis. J Agric Food Chem 53:3867-3871

98. Serpa R, Franc EJG, Furlaneto-maia L, Andrade CGTJ, Diniz A, Furlaneto MC (2012) In vitro antifungal activity of the flavonoid baicalein against Candida species. J Med Microbiol 61:1704-1708. https://doi.org/10.1099/jmm.0. 047852-0

99. Kanwala Q, Hussain I, Siddiqui HL, Javaid A (2010) Antifungal activity of flavonoids isolated from mango (Mangifera indica L.) leaves. Nat Prod Res 24(20):1907-1914. https://doi.org/10.1080/14786419.2010.488628

100. Vimala G, Shoba FG (2014) A review on antiulcer activity of few indian medicinal plants. Int J Microbiol 2014:1-14

\section{Publisher's Note}

Springer Nature remains neutral with regard to jurisdictional claims in published maps and institutional affiliations.

\section{Submit your manuscript to a SpringerOpen ${ }^{\circ}$ journal and benefit from:}

- Convenient online submission

- Rigorous peer review

- Open access: articles freely available online

- High visibility within the field

- Retaining the copyright to your article

Submit your next manuscript at $\boldsymbol{\nabla}$ springeropen.com 